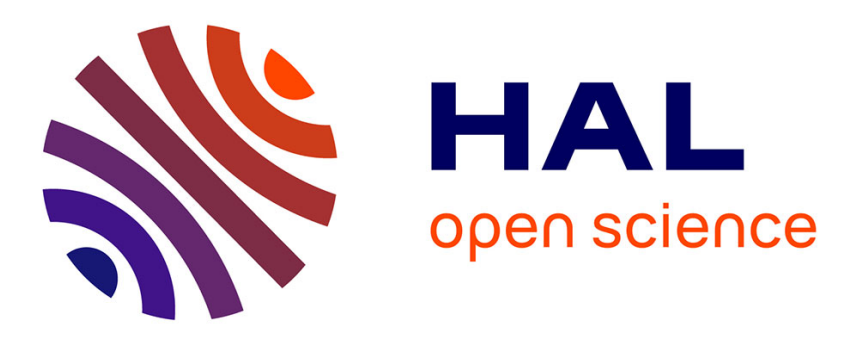

\title{
Robust Adaptive Tracking Control of Underwater Vehicles: Design, Stability Analysis and Experiments
}

\author{
Auwal Tijjani Shehu, Ahmed Chemori, Vincent Creuze
}

\section{To cite this version:}

Auwal Tijjani Shehu, Ahmed Chemori, Vincent Creuze. Robust Adaptive Tracking Control of Underwater Vehicles: Design, Stability Analysis and Experiments. IEEE/ASME Transactions on Mechatronics, 2021, 26 (2), pp.897-907. 10.1109/TMECH.2020.3012502 . lirmm-02918627

\section{HAL Id: lirmm-02918627 https://hal-lirmm.ccsd.cnrs.fr/lirmm-02918627}

Submitted on 20 Aug 2020

HAL is a multi-disciplinary open access archive for the deposit and dissemination of scientific research documents, whether they are published or not. The documents may come from teaching and research institutions in France or abroad, or from public or private research centers.
L'archive ouverte pluridisciplinaire HAL, est destinée au dépôt et à la diffusion de documents scientifiques de niveau recherche, publiés ou non, émanant des établissements d'enseignement et de recherche français ou étrangers, des laboratoires publics ou privés. 


\title{
Robust Adaptive Tracking Control of Underwater Vehicles: Design, Stability Analysis and Experiments
}

\author{
Auwal Shehu Tijjani, Member, IEEE, Ahmed Chemori, Senior Member, IEEE and Vincent Creuze
}

\begin{abstract}
The unpredictable nature of the marine environment, combined with nonlinear dynamics and parameter uncertainty of underwater vehicles makes the control system design for such vehicles a challenging task. Based on these issues, hybridising robustness and adaptation in the control system could result in more successful marine missions. This work proposes a robust adaptive control (RAC) scheme for trajectory tracking of an autonomous underwater vehicle. The proposed RAC scheme has been developed by exploiting the advantages of a robust sliding mode controller (SMC) and an adaptation law. Lyapunov arguments are proposed to prove the exponential stability and finite-time convergence of the resulting closed-loop dynamics tracking error to an invariant set, $S$ (very close to zero). Scenarios-based real-time experiments are conducted with the Leonard ROV prototype to demonstrate the effectiveness of the proposed RAC approach. The control design performance indices (root mean square error RMSE, integral absolute error IAE and integral square error ISE) and a comparative analysis with a recent control scheme from the literature confirm the interest of the proposed RAC scheme for marine applications.
\end{abstract}

Index Terms-Underwater vehicle, robust adaptive control, finite-time convergence, stability analysis, real-time experiments.

\section{INTRODUCTION}

$\mathbf{I}$ $\mathrm{N}$ the near future, underwater will provide an increasing part of the domestic, industrial as well as research resources, ranging from renewable energy to food for a better quality of human life on the land surface [1] [2]. Exploring and monitoring underwater environment is difficult, dangerous and expensive [3] [4] [5]. Accurate, cheap, autonomous and intelligent tools are required for exploiting such a challenging environment. Based on the aforementioned requirements, several autonomous underwater vehicles (AUVs) have been proposed by several research communities [6]; consequently, AUVs become a growing field of research, in the past decades [5] [6]. These vehicles have also potential applications in the military sector, ranging from maritime borders surveillance to minewarfare [7]. However, designing a control algorithm that guides the vehicle for a successful mission is one of the must challenging tasks [8]. This problem is mainly related to the parameters uncertainty and nonlinearity in the system dynamics, the hardware constraints and the unpredictable nature

Auwal Shehu Tijjani, Ahmed Chemori and Vincent Creuze are with the LIRMM, University of Montpellier, CNRS, Montpellier, France. (e-mail: atshehu@lirmm.fr; ahmed.chemori@lirmm.fr; vincent.creuze@lirmm.fr).

The authors acknowledged the Petroleum Technology Development Fund (PTDF), Nigeria, for first author Ph.D. financial support.

Manuscript received April 19, 2005; revised August 26, 2015. of the marine environment. As a consequence, the design of a tracking control scheme for the such vehicles becomes a critical issue [9]. Indeed, several control schemes have been proposed in the literature to address this problem, such as improved control schemes based on PD and PID control [10] [11] [12], robust control [13], adaptive control [14], intelligent and hybrid control [15].

Robust control is a wide area of research [14] [16], which includes sliding mode control (SMC). Since the control scheme proposed in this work is based on a modified SMC structure; we, hereafter review some of the recently proposed SMC control schemes from the literature. In [17], a high-order sliding mode control (HOSMC) was implemented on the Kaxan underwater vehicle as an improvement of the conventional SMC. Numerical simulations were used to demonstrate the effectiveness of the proposed control scheme. However, this controller has a high-frequency switching, and also, it requires the knowledge of the uncertainties bounds. Additionally, no real-time experiments are conducted. In a similar way, an improved SMC termed as double-loop sliding mode control (DSMC) has been proposed in [18]. The proposed DSMC was implemented by replacing the classical SMC switching term with an inverse tangent function to reduce the chattering phenomenon and the controller has been validated in simulation for trajectory tracking of a work-class underwater vehicle. The closed-loop system has been divided into a velocity and a position loop, and the performance was compared with a classical SMC and a fuzzy SMC. An improved PID fractionalorder fuzzy controller based on SMC structure, was reported in [19]; however, the validation scenarios proposed were tested only in simulation. Besides, an underwater vehicle is mainly equipped with thrusters actuated with electrical motors [20]. Being one of the crucial hardware parts of the vehicle, a thruster fault tolerance quantized SMC has been investigated in [21]. The proposed control approach has been implemented on an unmanned surface vehicle (USV) and shows a promising performance, which could be easily extended to underwater vehicles. Similarly, nonlinear fully actuated tracking control of underwater vehicles has been proposed in [22]. In this work, a velocity transformation has been proposed during tracking by decomposing the inertial matrix of the system. Tracking error leads to a variation in the decomposed inertial matrix; this variation is compensated in the feedback gain matrix, which updates the control signal. Consequently, this control scheme can be considered similar to adaptive control.

In marine applications, control schemes based on SMC 
structure can ensure robustness for trajectory tracking on underwater vehicles. For this reason, some research communities has exploited the robustness of the SMC, while trying to compensate for its drawbacks. Based on this notion, a deterministic policy gradient non-model-based learning algorithm SMC has been proposed and implemented on an autonomous underwater vehicle in [23]. However, the stability of the resulting closedloop system has not been proven. A type-2 robust adaptive fuzzy SMC has been reported in [24], which has a short rise time; but, its performance degrades when the vehicle changes its orientation. In [25], the robustness of a classical SMC toward parameter uncertainties for trajectory tracking of an autonomous underwater vehicle has been investigated. However, the proposed approach suffers from slow finite-time convergence on yaw tracking as the ratio of the uncertainty increases. Also, robust adaptive control schemes have been proposed in [26] and [27], both requiring velocity measurement. In practice, velocity measuring sensors (e.g. Doppler velocity logger, DVL) are quite expensive and most of the underwater vehicles are not equipped with such sensors. To avoid the need of velocity measurement, a nonlinear SMC neural networkbased controller has been investigated in [28]. Additionally, the neural network in this control scheme aims at adding some intelligent functionality to the proposed scheme, while reducing the computational cost associated with the classical neural network structure. An improved robust adaptive fuzzy neural with active disturbance rejection control scheme has been proposed in [29]. Experimentally validated, an adaptive fuzzy backstepping control has been proposed in [30]. Even though reasonable tracking error reduction was achieved, further improvement could be possible, especially when the two separate master-slave controllers communicate to effectively estimate the uncertain parameters. In [31], a backstepping SMC based on an adaptive disturbance observer has been proposed and implemented on an underwater vehicle for wall's crack detection. Robust feedback linearization control and perturbations attenuation based on a structure regulator have been proposed in [32] and [33] respectively; however, the linearization and attenuation could lead to the suppression of many interesting nonlinear behaviours. So far, most of the proposed control algorithms lack practical implementation prospects due to their complexity, implantation costs, as well as hardware resources limitation. Hence, classical PD and PID remained the dominating control schemes for real-time control of the underwater vehicles in many marine applications [12] [34].

Inspired by the real-time implementation simplicity of classical control such as PD, PID and the SMC proposed in [35], we exploit the robustness and adaptation to propose a robust adaptive controller (RAC) for tracking control of autonomous underwater vehicles. The proposed RAC scheme takes into consideration the nonlinearities, uncertainties and coupled dynamics of the vehicle, as well as the real-time feasibility and the uncertainty of the marine environment; thus, the contributions of this work are as follows:

(1) We proposed a RAC control scheme as a less computationally demanding robust scheme for tracking control of autonomous underwater vehicles. In the real-time implemen-

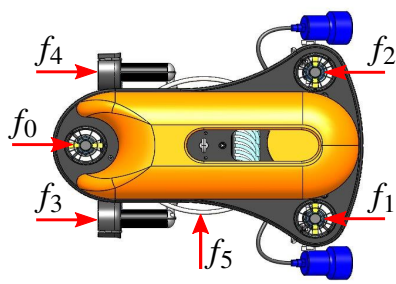

Fig. 1. CAD view of Leonard underwater vehicle with its thrusters configuration, which generated forces for transnational (surge, sway and depth) and rotational (roll, pitch and yaw) motions.

tation of the proposed RAC, we replace the discontinuous sign function of the SMC structure proposed in [35] with a continuous function, thereby solving the chattering problem described in [35]. Also, a continuous function-based adaptation law is used to avoid overestimation of the controller gains and to decrease chattering. The proposed RAC does not require the knowledge of the uncertainties. Additionally, it can be implemented in real-time on underwater vehicles without DVL, contrary to many of the proposed schemes in the literature.

(2) Lyapunov arguments are used to prove the exponential stability of the resulting closed-loop dynamics of the vehicle using the proposed RAC scheme, which guarantee a stable behaviour for real-time marine applications. Moreover, a finitetime convergence of the proposed scheme is assured. Contrary to the majority of the control schemes proposed in the literature, where only an asymptotic convergence is guaranteed.

(3) Different real-time scenarios are conducted and compared to validate the effectiveness and robustness of the proposed scheme.

(4) Also in this work, the parameters tuning process of the proposed RAC scheme is detailed.

The remaining parts of this paper are organised as follows: Section II describes the technical features of the vehicle (Leonard), as well as its modelling. The proposed RAC law and stability analysis are addressed in sections III and IV, respectively. Scenarios-based real-time experimental results are detailed and discussed in section V. Section VI finalises the paper sections with the conclusion and future work.

\section{Vehicle Description AND MOdElling}

In this section, we describe the main features, as well as the dynamic modelling of the vehicle used for implementing the proposed robust adaptive control scheme.

\section{A. Vehicle Description}

The real-time experiments are conducted using one of the LIRMM's underwater vehicle prototypes, called Leonard, illustrated in Fig. 11. The vehicle is fully actuated (i.e. a holonomic system) in all its six degrees of freedom (6 DOFs). The vehicle can either be remotely operated by a pilot or controlled by an algorithm to carry out tasks autonomously. Hence, Leonard ROV can be operated as a completely autonomous underwater or in shared control. In this work, the real-time experiments are conducted with the vehicle operated 
TABLE I

TECHNICAL SPECIFICATIONS OF LEONARD ROV

\begin{tabular}{|l|l|}
\hline Components & Specifications/Descriptions \\
\hline Attitude Sensor & Sparkfun MPU 9250, MEMS 9-axes gyrometer, \\
& accelerometer and magnetometer microprocessor. \\
Cell Latitude E6230 Intel Core i7 - 2.9 GHz & Windows 7 Professional 64 bits Microsoft \\
& Visual C++ 2010. \\
Depth Sensor & Pressure Sensor MS5803-14BA. \\
Dimensions & Length $=75 \mathrm{~cm}$, width $=55 \mathrm{~cm}$ and height $=45 \mathrm{~cm}$. \\
Floatability & $9 \mathrm{~N}$. \\
Light & $50 \mathrm{~W}$ LED. \\
Mass & 28kg. \\
Maximal Depth & $100 \mathrm{~m}$. \\
Power Consumption & $48 \mathrm{~V}, 600 \mathrm{~W}$. \\
Sampling Period & $50 \mathrm{~ms}$. \\
Tether & $150 \mathrm{~m}$. \\
Thrusters & 6-Seabotix BTD150 continuous thrust $2.2 \mathrm{kgf}$ each \\
& with Syren 10 drivers. \\
\hline
\end{tabular}

as a completely autonomous underwater vehicle. According to [10], [11] and [14], several marine missions from inspection to intervention operations require the roll $(\phi)$ and the pitch $(\theta)$ of the underwater vehicle to be maintained around zero (i.e. horizontally). In the design of Leonard ROV, the positions of the centre of buoyancy and the centre of gravity make the robot passively stable in pitch and roll. This feature helps to minimise the energy consumption of the vehicle. However, the autonomous control algorithm proposed in this work is designed to stabilise the vehicle at any desired orientation. The vehicle's technical specifications are summarised in TABLE[

\section{B. Vehicle Modelling}

The mathematical description of the vehicle in 6 DOFs is categorised into kinematics and dynamics. Two unique frames of references are assigned for the vehicle navigation as illustrated in Fig. 2. These frames assignment are based on SNAME convention [4] [10] [14]. The frames are :

1) Inertial or Earth-fixed reference frame, usually at the water surface.

2) Vehicle or body-fixed reference frame, generally coinciding with the centre of volume of the vehicle.

The vehicle is modelled as follows:

1) Leonard ROV Kinematics: Let us define the inertial (Earth-fixed) reference frame as $R_{n}$ and the body-fixed reference frame $R_{b}$, as illustrated in Fig. 2 Then, we can relate the rate of change of position and orientation of the vehicle in $R_{n}$ with linear and angular velocities of the vehicle in $R_{b}$ using the formular:

$$
\dot{\eta}=J(\eta) v
$$

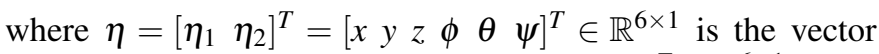
of position and orientation, $v=\left[\begin{array}{lllll}u & v & w & p & q\end{array}\right]^{T} \in \mathbb{R}^{6 \times 1}$ is the linear and angular velocities vector and $J(\eta) \in \mathbb{R}^{6 \times 6}$ defines the transformation from $R_{n}$ to $R_{b}$ [36].

2) Leonard ROV Dynamics: In accordance with the SNAME convention [36], the 6-DOF dynamics of the vehicle in the body-fixed frame $R_{b}$, is expressed by (2):

$$
M \dot{v}+C(v) v+D(v) v+g(\eta)=\tau+w^{\star}(t)
$$

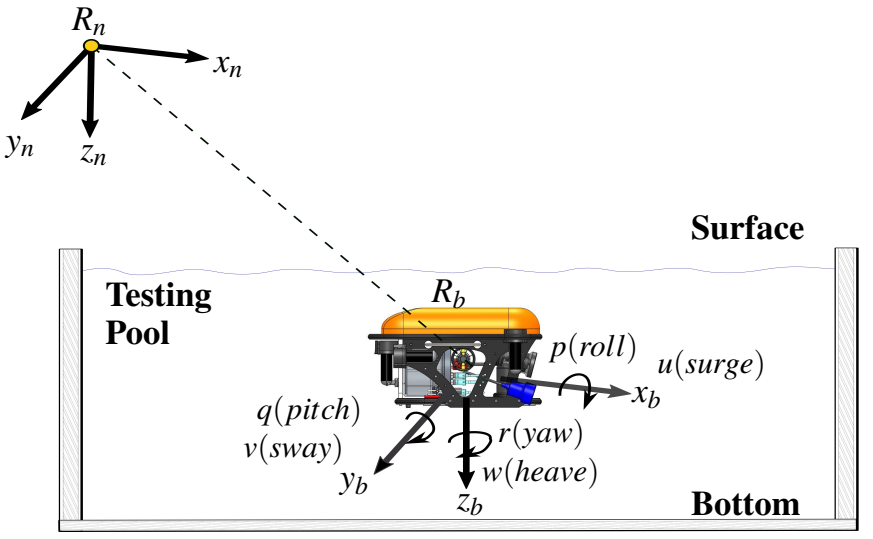

Fig. 2. Body-fixed reference frame $R_{b}$ and inertial fixed reference frame $R_{n}$ assigned to Leonard ROV for kinematic and dynamic modelling.

where $M \in \mathbb{R}^{6 \times 6}$ is the inertia matrix, $C(v) \in \mathbb{R}^{6 \times 6}$ is the Coriolis and centripetal matrix, $D(v) \in \mathbb{R}^{6 \times 6}$ is the hydrodynamic damping and lift matrix, $g(\eta) \in \mathbb{R}^{6 \times 1}$ is the vector of restoring forces and moments, $\tau \in \mathbb{R}^{6 \times 1}$ is the vector of control inputs and $w^{\star}(t) \in \mathbb{R}^{6 \times 1}$ is the time varying disturbance vector.

Based on (2), the details of the terms describing the dynamics are given as follows.

The inertia matrix $\mathrm{M}$ of the vehicle, comprises the inertia of the added mass $M_{A}$ and rigid-body inertia $M_{R B}$ that is:

$$
M=M_{R B}+M_{A}
$$

The matrix $\mathrm{M}$ has the following property:

Property P1: This matrix $M$ is bounded and symmetric positive definite, when an underwater vehicle is moving slowly [36] [37], i.e. there exist positive constants $\kappa_{1}, \kappa_{2}$ such that $M$ satisfies: $0<\kappa_{1} I \leq M=M^{T} \leq \kappa_{2} I<\infty$, where $I$ is an identity matrix having the same dimension as $M$.

Similarly, the matrix $C(v)$ defined in 2 has the following property:

Property P2: The parameterisation of matrix $C(v)$ is always possible such that it is skew-symmetric [36] [37], i.e.

$$
C(v)=-C^{T}(v), \forall v \in \mathbb{R}^{6 \times 1}
$$

Another term worth to be discussed in the vehicle's dynamics given in 2 is the damping matrix $D(v)$, which is approximately defined in $R_{b}$ as follows:

$$
D(v)=\operatorname{diag}\left\{X_{u}, Y_{v}, Z_{w}, K_{p}, M_{q}, N_{r}\right\}
$$

where $\left\{X_{u}, Y_{v}, Z_{w}, K_{p}, M_{q}, N_{r}\right\}$ are the hydrodynamic elements. The numerical value of the matrix $D(v)$ of the vehicle has been experimentally determined (see APPENDIX A-A [10]).

The term $g(\eta)$ is a vector of restoring forces and moments, produced by the buoyancy force $f_{B}$ and the weight $f_{W}$ of the vehicle. $f_{B}$ and $f_{W}$ act along the z-axis with reference to $R_{n}$. The transformation of $f_{B}$ and $f_{W}$ to $R_{b}$ yields the restoring 
forces and moments (see APPENDIX A-B). Thus, $g(\eta)$ is given by:

$$
g(\eta)=\left[\begin{array}{c}
F_{g(\eta)} \\
M_{g(\eta)}
\end{array}\right]=\left[\begin{array}{c}
f_{B} s \theta \\
-f_{B} c \theta s \psi \\
-f_{B} c \theta c \psi \\
-r(z)_{c b} B c \theta s \psi \\
-r(z)_{c b} B s \theta \\
0
\end{array}\right]
$$

Finally, $\tau$ is the vehicle control input signal. The control signal is a vector of forces (producing surge $\mathrm{u}$, sway $\mathrm{v}$ and heave $\mathrm{w}$, as illustrated in Fig. 2) and torques (producing roll p, pitch $\mathrm{q}$ and yaw r, as also illustrated in Fig. 2) generated by the vehicle's thrusters $\left(f_{0}, f_{1}, f_{2}, f_{3}, f_{4}\right.$ and $f_{5}$ whose configuration is illustrated in Fig. 11). Thus, $\tau$ is given in a compact vector form as:

$$
\tau=T \cdot f
$$

where $T \in \mathbb{R}^{6 \times 6}$ is the thrusters' configuration matrix and $f=\left[\begin{array}{llllll}f_{0} & f_{1} & f_{2} & f_{3} & f_{4} & f_{5}\end{array}\right]^{T}$ represent the forces produced by the six thrusters of the vehicle. The hypothesis 1 and 2 hereafter are taken into account within control system design. Hypothesis 1: The thrusters' velocity of an underwater vehicle is limited by its actuators' saturation. Thus, the Leonard ROV thrusters' velocities are upper bounded by positive constants.

Hypothesis 2: The disturbance $w^{\star}(t)$ defined in (2) is assumed to be a Lipschitz continuous function bounded by Lipschitz constant.

\section{Proposed Robust Adaptive Control Law}

In this section, we present the design of the proposed Robust Adaptive Control (RAC) law for trajectory tracking. This control scheme is inspired from the control structure proposed in [35]. The controller proposed in [35] has some significant advantages such as robustness, model-free structure, and unnecessary knowledge of the uncertainties. However, the authors used discontinuous functions in both the control structure and adaptation law; this produces undesired chattering phenomenon, which may damage the actuators. Moreover, the scheme was implemented on a nonlinear uncertain singleinput single-output (SISO) system and validated only through numerical simulations. We proposed to improve this scheme using a smooth approximation through a hyperbolic tangent function instead of the discontinuous function in both the control structure and the adaptation law, to decrease the chattering problem. However, for a rigorous theory of the proposed RAC scheme and its stability analysis, we use the sign function. Additionally, we modified the structure of the adaptation law using a different function to avoid an overestimation of the adaptive gains. Also, we extend the scheme to the uncertain coupled multiple-input multiple-out (MIMO) nonlinear systems representing underwater vehicles. Finally, the improved proposed RAC scheme in this work is implemented on Leonard ROV and validated through realtime experiments, using different scenarios. The conducted experiments reveal the effectiveness of the proposed RAC in terms of robustness toward parameters uncertainties and external disturbance rejection for real-time applications. These qualities of the proposed RAC scheme qualifies it to be a possible candidate for solving the tracking problem of underwater vehicles; despite the uncertainty in the vehicles dynamics and unpredictable nature of the marine environment. The proposed RAC law is designed in the sequel.

Let us first consider,

$$
\begin{gathered}
\eta_{d}(t)=\left[x_{d}(t), y_{d}(t), z_{d}(t), \phi_{d}(t), \theta_{d}(t), \psi_{d}(t)\right]^{T} \\
\eta(t)=[x(t), y(t), z(t), \phi(t), \theta(t), \psi(t)]^{T}
\end{gathered}
$$

as the desired and actual vehicle's trajectories respectively.

The corresponding trajectory tracking error $e(t)$ can be written as follows:

$$
e(t)=\eta_{d}(t)-\eta(t)
$$

where $e(t)=\left[e_{1}(t), e_{2}(t), \ldots, e_{6}(t)\right]^{T}$, while $\eta_{d}(t)$ and $\eta(t)$ are as defined in (8) and (9) respectively.

At this point, we can compute the first time derivative of (10), which yields:

$$
\dot{e}(t)=\dot{\eta}_{d}(t)-\dot{\eta}(t)
$$

where $\dot{e}(t)=\left[\dot{e}_{1}(t), \dot{e}_{2}(t), \ldots, \dot{e}_{6}(t)\right]^{T}$, while $\dot{\eta}_{d}(t)$ and $\dot{\eta}(t)$ are the time derivative of $(8)$ and $(9)$ respectively.

Now, it is possible to define the sliding surface using $e(t)$ and $\dot{e}(t)$ as follows:

$$
\sigma(t)=\dot{e}(t)+B e(t)
$$

where $\sigma(t)=\left[\sigma_{1}, \sigma_{2}, \ldots, \sigma_{6}\right]^{T}$, and $B=\operatorname{diag}\left\{\beta_{1}, \beta_{2}, \ldots, \beta_{6}\right\}$, is a positive definite diagonal matrix, which modifies the convergence (rate) of $\dot{e}(t)$ to the origin (when, $\sigma(t)=0$ ).

The control signals for all the 6 DOFs of the underwater vehicle are designed as follows:

$$
\tau(\eta)=\Lambda(t) \operatorname{sgn}[\sigma(t)]
$$

where $\tau(\eta)$ represents vector of the control inputs of the vehicle, $\Lambda(t)=\operatorname{diag}\left\{\Lambda_{1}, \Lambda_{2}, \ldots, \Lambda_{6}\right\}$ is the adaptive gain matrix with positive definite diagonal elements, $\operatorname{sgn}[$.$] represents the$ standard discontinuous signum function and $\sigma(t)$ has been defined in (12).

From (13), each diagonal element $\Lambda_{i}$ (with $i=\overline{1,6}$ ) of the matrix $\Lambda(t)$ is updated using an adaptation law designed as follows:

$$
\dot{\Lambda}_{i}= \begin{cases}\Gamma_{i} \cdot\left|\sigma_{i}\right| \cdot \operatorname{sgn}\left[\left|\sigma_{i}\right|-\mu_{i}\right], & \text { if } \Lambda_{i}>\lambda_{i} \\ \gamma_{i}, & \text { if } \Lambda_{i}=\lambda_{i} \\ \lambda_{i}, & \text { if } \Lambda_{i}<\lambda_{i}, i=\overline{1,6}\end{cases}
$$

where $\Lambda_{i}>0$ is an adaptive positive gain, $\Gamma_{i}>0$ is an adaptation rate, $\lambda_{i}>0$ is the minimum value of the adaptive gain, $\gamma_{i}>0$ is a positive design parameter, $\mu_{i}>0$ is a positive design parameter determining the loss of robustness, $\sigma(t)$ has been defined in 12 as the sliding surface, $\dot{\Lambda}(0)>0$ is the initial condition of the adaptation dynamics.

Remark 1: The desired trajectory $\eta_{d}(t)$, as well as its first and second time derivatives are assumed to be smooth and bounded. 


\section{STABility ANALYSis}

In this section, the exponential stability and finite-time convergence of the underwater vehicle closed-loop dynamics to the desired trajectory in real-time marine applications are addressed analytically. To simplify the ROV's closed-loop stability analysis, equation (2) is transformed (see APPENDIX A-C) and defined in $R_{n}$ as follows [36]:

$$
\bar{M}(\eta) \ddot{\eta}+\bar{C}(v, \eta) \dot{\eta}+\bar{D}(v, \eta) \dot{\eta}+\bar{g}(\eta)=\bar{\tau}(\eta)+\bar{w}^{\star}(t)
$$

where all the terms in (15) have been defined in (2) before transforming to $R_{n}$.

Theorem 1: The proposed controller defined by (13) and the adaptation dynamics (14) ensure that the trajectory (9) of the uncertain coupled nonlinear MIMO dynamics subject to external disturbance and defined in (15) is bounded and converges exponentially to the desired trajectory (8) in a finitetime $t_{f}$ (and remains in (8) $\forall t \geq t_{f}$ ); as long as the proposed RAC gains are selected sufficiently large enough based on the initial condition of the vehicle's states, while the parameter $\rho$ satisfies the following conditions:

$$
\rho>\frac{\|\Gamma\|\|\sigma\|\left[\Lambda-\Lambda^{\star}\right]^{T}[\cdot]}{\Omega_{b}\|\sigma\|\left[\|\Lambda\|-\left\|\Lambda^{\star}\right\|\right]-\Lambda_{k}\left\|\Lambda-\Lambda^{\star}\right\|}
$$

where $[\cdot]=\left[\operatorname{sgn}\left[\left|\sigma_{1}\right|-\mu_{1}\right], \operatorname{sgn}\left[\left|\sigma_{2}\right|-\mu_{2}\right], \ldots, \operatorname{sgn}\left[\left|\sigma_{6}\right|-\mu_{6}\right]\right]^{T}$ for Condition 1; $[\cdot]=\left[\gamma_{1}, \gamma_{2}, \ldots, \gamma_{6}\right]^{T}$ for Condition 2; and $[\cdot]=\left[\lambda_{1}, \lambda_{2}, \ldots, \lambda_{6}\right]^{T}$ for Condition 3 (see the details of the analysis below). All the parameters $\sigma_{i}, \mu_{i}, \gamma_{i}, \lambda_{i}, \Gamma_{i}, \Lambda_{i}$ have been defined in the previous section, while $\rho, \Omega_{b}, \Lambda_{k}, \Lambda^{\star}$ are defined accordingly in the subsequent proof of the stability.

Proof: Let us consider the Lyapunov candidate function $V: \mathbb{R}^{n \times 1} \rightarrow \mathbb{R}(n \in[1,2, \ldots, 6])$, positive definite, Lipschitz continuous function, defined as:

$$
V(\sigma, \tilde{\Lambda})=\frac{1}{2} \sigma^{T} \sigma+\frac{1}{2 \rho} \tilde{\Lambda}^{T} \tilde{\Lambda}
$$

and $\quad$ satisfying $\quad \Lambda_{\alpha k 1}\left[\|\sigma\|^{2}+\frac{\|\tilde{\Lambda}\|^{2}}{\rho}\right]^{1 / 2} \leq V(\sigma, \tilde{\Lambda}) \leq$ $\Lambda_{\alpha k 2}\left[\|\sigma\|^{2}+\frac{\|\tilde{\Lambda}\|^{2}}{\rho}\right]^{1 / 2}$, where $\Lambda_{\alpha k 1}$ and $\Lambda_{\alpha k 2}$, are positive constants, $\Lambda$ and $\Lambda^{\star}$ are the estimated adaptive positive definite gain and the positive constant upper bound, respectively and $\rho$ is a positive design parameter (i.e. $\rho>0$ ). Additionally, $\sigma=\left[\sigma_{1}, \sigma_{2}, \ldots, \sigma_{6}\right]^{T}$ has been defined in 12 and $\tilde{\Lambda}$, is the difference between the estimated adaptive positive definite gain $(\Lambda)$ and the positive constant upper bound $\left(\Lambda^{\star}\right)$, i.e. $\tilde{\Lambda}=\Lambda-\Lambda^{\star}, \quad \Lambda=\left[\Lambda_{1}, \Lambda_{2}, \ldots, \Lambda_{6}\right]^{T}$ and $\Lambda^{\star}=\left[\Lambda_{1}^{\star} \Lambda_{2}^{\star}, \ldots, \Lambda_{6}^{\star}\right]^{T}$.

Remark 2: Since the positive adaptive gain $\Lambda$ is not allowed to grow indefinitely large (to avoid overestimation) and is upper bounded by positive constant $\Lambda^{\star}$, then $\tilde{\Lambda}<0$.

To further facilitate the investigation of the remaining properties of the proposed Lyapunov candidate function, defined in (16), let us differentiate (11) and (12) respectively, as follows:

$$
\begin{aligned}
& \ddot{e}(t)=\ddot{\eta}_{d}(t)-\ddot{\eta}(t) \\
& \dot{\sigma}(t)=\ddot{e}(t)+B \dot{e}(t)
\end{aligned}
$$

where all the terms in (17) and (18) have been defined in (11) and (12) respectively.
Taking the time derivative of the proposed Lyapunov candidate function in 16 yields:

$$
\dot{V}(\sigma, \tilde{\Lambda})=\sigma^{T} \dot{\sigma}+\frac{1}{\rho} \tilde{\Lambda}^{T} \dot{\tilde{\Lambda}}
$$

Substituting (17), as well as the vehicle's dynamics 15 into (18), one obtains:

$$
\begin{array}{r}
\dot{\sigma}(t)=\ddot{\eta}_{d}(t)-[\bar{M}(\eta)]^{-1}\left[\bar{\tau}(\eta)+\bar{w}^{\star}(t)-\bar{C}(\nu, \eta) \dot{\eta}\right. \\
-\bar{D}(v, \eta) \dot{\eta}-\bar{g}(\eta)]+B \dot{e}(t)
\end{array}
$$

Assumption A1 : In real-time experiments, the Leonard ROV moves slowly. Thus, the effect of Coriolis and centripetal forces is assumed to be negligible [4].

Invoking assumption $\mathbf{A 1}$ above, equation $(20)$ becomes:

$$
\begin{array}{r}
\dot{\sigma}(t)=\ddot{\eta}_{d}(t)-[\bar{M}(\eta)]^{-1} \bar{\tau}(\eta)-[\bar{M}(\eta)]^{-1}\left[\bar{w}^{\star}(t)\right. \\
-\bar{D}(v, \eta) \dot{\eta}-\bar{g}(\eta)]+B \dot{e}(t)
\end{array}
$$

From (21), let us introduce $\Phi$, defined as follows:

$$
\begin{array}{r}
\Phi=[\bar{M}(\eta)]^{-1}\left[\bar{w}^{\star}(t)-\bar{D}(v, \eta) \dot{\eta}-\bar{g}(\eta)\right]-B \dot{e}(t)-\ddot{\eta}_{d}(t), \\
\text { and } \Omega \text { defined as } \Omega=[\bar{M}(\eta)]^{-1}
\end{array}
$$

Now, we can simplify (21) using (22) as follows:

$$
\dot{\sigma}(t)=-[\Phi+\Omega \bar{\tau}(\eta)]
$$

Substituting 23 and $\tilde{\Lambda}=\Lambda-\Lambda^{\star}$ into $(19)$ leads to:

$$
\dot{V}(\sigma, \tilde{\Lambda})=-\sigma^{T}[\Phi+\Omega \bar{\tau}(\eta)]+\frac{1}{\rho}\left[\Lambda-\Lambda^{\star}\right]^{T} \dot{\Lambda}
$$

Injecting the proposed control law (13) into (24), yields:

$$
\dot{V}(\sigma, \tilde{\Lambda})=-\sigma^{T}[\Phi+\Omega \Lambda \operatorname{sgn}[\sigma]]+\frac{1}{\rho}\left[\Lambda-\Lambda^{\star}\right]^{T} \dot{\Lambda}
$$

Assumption A2: Based on property $P 1, M$ is symmetric positive definite and bounded matrix, therefore $M^{-1}$ exists and is also positive definite [38] [39]. This implies that $\bar{M}(\eta)$ is symmetric positive definite and bounded, also $\bar{M}(\eta)]^{-1}$ exists and is positive definite [36] [38] [39]. Similarly, $\bar{D}(v, \eta)$ is positive definite (see [36] [37] [39] ) and $\bar{g}(\eta)$ is bounded (see [39]). Hence, we can assume that $\|\Phi\|$ and $\|\Omega\|$, defined in (22), are bounded by positive constants $\Phi_{b}$ and $\Omega_{b}$, respectively.

Based on assumption $\mathbf{A 2}$ above, 25] can be rewritten as:

$$
\dot{V}(\sigma, \tilde{\Lambda}) \leq-\Phi_{b}\|\sigma\|-\Omega_{b}\|\Lambda\|\|\sigma\|+\frac{1}{\rho}\left[\Lambda-\Lambda^{\star}\right]^{T} \dot{\Lambda}
$$

To introduce the adaptation law defined in (14) into 26, the following conditions are possible:

Condition 1: If $\Lambda_{i}>\lambda_{i}$ for each DOF of the vehicle then:

$$
\dot{\Lambda}_{i}=\Gamma_{i} \cdot\left|\sigma_{i}\right| \cdot \operatorname{sgn}\left[\left|\sigma_{i}\right|-\mu_{i}\right], \quad i=\overline{1,6}
$$


where $\dot{\Lambda}=\left[\dot{\Lambda}_{1}, \dot{\Lambda}_{2}, \ldots, \dot{\Lambda}_{6}\right]^{T}$, adaptation dynamics defined in (14).

Substituting (27) into 26) for each DOF leads to:

$$
\begin{array}{r}
\dot{V}(\sigma, \tilde{\Lambda})=-\Phi_{b}\|\sigma\|-\Omega_{b}\left\|\Lambda^{\star}\right\|\|\sigma\|+\Omega_{b}\left\|\Lambda^{\star}\right\|\|\sigma\| \\
-\Omega_{b}\|\Lambda\|\|\sigma\|+\frac{1}{\rho}\|\Gamma\|\|\sigma\|[\Lambda \\
\left.\left.-\Lambda^{\star}\right]^{T}\left[\operatorname{sgn}\left[\left|\sigma_{1}\right|-\mu_{1}\right], \operatorname{sgn}\left[\left|\sigma_{2}\right|-\mu_{2}\right], \ldots, \operatorname{sgn} \| \sigma_{6} \mid-\mu_{6}\right]\right]^{T}
\end{array}
$$

Introducing $\Lambda_{k}>0$ and $\Lambda_{\alpha}=\Phi_{b}+\Omega_{b}\left\|\Lambda^{\star}\right\|$, where $\Lambda_{\alpha}>$ 0 (since $\Phi_{b}+\Omega_{b}\left\|\Lambda^{\star}\right\|>0$ ). Thus, 28) can be rewritten as follows:

$$
\begin{array}{r}
\dot{V}(\sigma, \tilde{\Lambda})=-\Lambda_{\alpha}\|\sigma\|-\Lambda_{k}\left\|\Lambda-\Lambda^{\star}\right\|-\left[\Omega_{b}\|\sigma\|\left[\|\Lambda\|-\left\|\Lambda^{\star}\right\|\right]\right. \\
-\Lambda_{k}\left\|\Lambda-\Lambda^{\star}\right\|-\frac{1}{\rho}\|\Gamma\|\|\sigma\|[\Lambda \\
\left.\left.-\Lambda^{\star}\right]^{T}\left[\operatorname{sgn}\left[\left|\sigma_{1}\right|-\mu_{1}\right], \operatorname{sgn}\left[\left|\sigma_{2}\right|-\mu_{2}\right], \ldots, \operatorname{sgn}\left[\left|\sigma_{6}\right|-\mu_{6}\right]\right]^{T}\right]
\end{array}
$$

Then, we can rewrite (29) as follows:

$$
\dot{V}(\sigma, \tilde{\Lambda})=-\Lambda_{\alpha}\|\sigma\|-\Lambda_{k}\left\|\Lambda-\Lambda^{\star}\right\|-\varsigma
$$

where,

$\varsigma=\Omega_{b}\|\sigma\|\left[\|\Lambda\|-\left\|\Lambda^{\star}\right\|\right]-\Lambda_{k}\left\|\Lambda-\Lambda^{\star}\right\|-\frac{1}{\rho}\|\Gamma\|\|\sigma\|[\Lambda$

$\left.\left.\left.-\Lambda^{\star}\right]^{T}\left[\operatorname{sgn}\left[\left|\sigma_{1}\right|-\mu_{1}\right], \operatorname{sgn} \llbracket\left|\sigma_{2}\right|-\mu_{2}\right], \ldots, \operatorname{sgn} \llbracket\left|\sigma_{6}\right|-\mu_{6}\right]\right]^{T}$

Hence, from $30 \hat{V}(\sigma, \tilde{\Lambda})$ becomes:

$$
\dot{V}(\sigma, \tilde{\Lambda}) \leq-\min \left\{\Lambda_{\alpha} \sqrt{2}, \Lambda_{k} \sqrt{2 \rho}\right\}\left[\frac{\|\sigma\|}{\sqrt{2}}+\frac{\left\|\Lambda-\Lambda^{\star}\right\|}{\sqrt{2 \rho}}\right]-\varsigma
$$

We can now express 31 in exponential form as:

$$
\dot{V}(\sigma, \tilde{\Lambda}) \leq-\Lambda_{\alpha k} \cdot V(\sigma, \tilde{\Lambda})^{1 / 2}-\varsigma
$$

where $\Lambda_{\alpha k}=\min \left\{\Lambda_{\alpha} \sqrt{2}, \Lambda_{k} \sqrt{2 \rho}\right\}$.

From (32) to conclude the exponential and finite-time convergence of $\|\sigma\|$ (when $\left|\sigma_{i}\right|>\mu_{i}, i=\overline{1,6}$ ) to a domain $\|\sigma\| \leq\|\mu\|$, we need to ensure that, in any case, $\varsigma>0$. For $\varsigma>0, \rho$ should be designed to satisfy the following inequality:

$$
\rho>\frac{\|\Gamma\|\|\sigma\|\left[\Lambda-\Lambda^{\star}\right]^{T}[\cdot]}{\Omega_{b}\|\sigma\|\left[\|\Lambda\|-\left\|\Lambda^{\star}\right\|\right]-\Lambda_{k}\left\|\Lambda-\Lambda^{\star}\right\|}
$$

where $[\cdot]=\left[\operatorname{sgn}\left[\left|\sigma_{1}\right|-\mu_{1}\right], \operatorname{sgn}\left[\left|\sigma_{2}\right|-\mu_{2}\right], \ldots, \operatorname{sgn}\left[\left|\sigma_{6}\right|-\mu_{6}\right]\right]^{T}$ In a situation when the scalar $\left|\sigma_{i}\right|=\mu_{i}, i=\overline{1,6}$ for each DOF, then it is sufficient to select $\Lambda_{k}$ as:

$$
\Lambda_{k}<\frac{\|\sigma\| \Omega_{b}\left[\|\Lambda\|-\left\|\Lambda^{\star}\right\|\right]}{\left\|\Lambda-\Lambda^{\star}\right\|}
$$

However, in a situation where $\left|\sigma_{i}\right|<\mu_{i}, i=\overline{1,6}$, for each DOF, it is possible that $\varsigma<0$ in 32 ; then, $\dot{V}(\sigma, \tilde{\Lambda})$ may be signed indefinite. As a consequence, exponential convergence of $\|\sigma\|$ in finite-time is only guaranteed, if the adaptation grows faster such that $\left|\sigma_{i}\right| \leq \mu_{i}, i=\overline{1,6}$. Exponential convergence of $\|\sigma\|$ in finite-time is guaranteed as soon as $\left|\sigma_{i}\right| \leq \mu_{i}, i=\overline{1,6}$ is established, while $V(\sigma, \tilde{\Lambda})$ starts decreasing, which leads to $\left|\sigma_{i}\right|<\mu_{i}, i=\overline{1,6}$ again. This phenomenon causes an overshoot, which can be evaluated (see [35]) as:

$$
\delta_{i}=\sqrt{\mu_{i}^{2}+\frac{\Phi_{b}^{2}}{\Gamma_{i} \Omega_{b}}}, i=\overline{1,6}
$$

where $\delta_{i}>0$, while $\mu_{i}, \Gamma_{i}$, are defined in (14) and $\Phi_{b}, \Omega_{b}$ are defined in assumption A2. Thus, $\|\sigma\|$ converges exponentially to a slightly bigger domain $\|\sigma\| \leq\|\delta\|$ in finite-time.

Condition 2: If $\Lambda_{i}=\lambda_{i}$ for each DOF of the vehicle then:

$$
\dot{\Lambda}_{i}=\gamma_{i}, \quad i=\overline{1,6}
$$

where $\dot{\Lambda}$, is the adaptation dynamics (see 277) and $\gamma_{i}$ is defined in (14).

Similarly, substituting [36, for each DOF, in $\varsigma$ of 32 yields:

$$
\dot{V}(\sigma, \tilde{\Lambda}) \leq-\Lambda_{\alpha k} \cdot V(\sigma, \tilde{\Lambda})^{1 / 2}-\varsigma
$$

where,

$$
\varsigma=\Omega_{b}\|\sigma\|\left[\|\Lambda\|-\left\|\Lambda^{\star}\right\|\right]-\Lambda_{k}\left\|\Lambda-\Lambda^{\star}\right\|-\frac{1}{\rho}\left[\Lambda-\Lambda^{\star}\right]^{T}\left[\gamma_{1}, \gamma_{2},\right.
$$

Hence, (37) is exponential stable if $\varsigma>0$. To ensure that, $\rho$ must satisfy the following inequality:

$$
\rho>\frac{\left[\Lambda-\Lambda^{\star}\right]^{T}\left[\gamma_{1}, \gamma_{2}, \ldots, \gamma_{6}\right]^{T}}{\Omega_{b}\|\sigma\|\left[\|\Lambda\|-\left\|\Lambda^{\star}\right\|\right]-\Lambda_{k}\left\|\Lambda-\Lambda^{\star}\right\|}
$$

When (38) is satisfied, we guarantee that $\|\sigma\|$ converges exponentially in finite-time to a domain $\|\sigma\| \leq\|\mu\|$.

Condition 3 : In the situation where $\Lambda_{i}<\lambda_{i}$, for each DOF of the vehicle, then:

$$
\dot{\Lambda}_{i}=\lambda_{i}, \quad i=\overline{1,6}
$$

where $\dot{\Lambda}$, is the adaptation dynamics (see 27p) and is $\lambda_{i}$ defined in (14).

Similarly, substituting (39), for each DOF, into $\varsigma$ of (32) yields:

$$
\dot{V}(\sigma, \tilde{\Lambda}) \leq-\Lambda_{\alpha k} \cdot V(\sigma, \tilde{\Lambda})^{1 / 2}-\varsigma
$$

where,

$\varsigma=\Omega_{b}\|\sigma\|\left[\|\Lambda\|-\left\|\Lambda^{\star}\right\|\right]-\Lambda_{k}\left\|\Lambda-\Lambda^{\star}\right\|-\frac{1}{\rho}\left[\Lambda-\Lambda^{\star}\right]^{T}\left[\lambda_{1}, \lambda_{2}\right.$, $\left.\ldots, \lambda_{6}\right]^{T}$

The argument 40 holds if $\varsigma>0$; to achieve this, $\rho$ must satisfy:

$$
\rho>\frac{\left[\Lambda-\Lambda^{\star}\right]^{T}\left[\lambda_{1}, \lambda_{2}, \ldots, \lambda_{6}\right]^{T}}{\Omega_{b}\|\sigma\|\left[\|\Lambda\|-\left\|\Lambda^{\star}\right\|\right]-\Lambda_{k}\left\|\Lambda-\Lambda^{\star}\right\|}
$$

When the argument (41) is satisfied, the exponential convergence of $\|\sigma\|$ in finite-time to a domain $\|\sigma\| \leq\|\mu\|$ is guaranteed.

Based on the conditions investigated above, $\dot{V}(\sigma, \tilde{\Lambda})$ satisfies (32), 37) and (40) when $\rho$ is properly designed using 33), (38) and 41), respectively. $V(\sigma, \tilde{\Lambda})$ is a positive definite, Lipschitz continuous function which satisfies $\Lambda_{\alpha k 1}\left[\|\sigma\|^{2}+\right.$ $\left.\frac{\|\tilde{\Lambda}\|^{2}}{\rho}\right]^{1 / 2} \leq V(\sigma, \tilde{\Lambda}) \leq \Lambda_{\alpha k 2}\left[\|\sigma\|^{2}+\frac{\|\tilde{\Lambda}\|^{2}}{\rho}\right]^{1 / 2}$. Hence, we can guarantee that $V(\sigma, \tilde{\Lambda})$ is exponentially stable, which implies 
that $\|\sigma\|$ converges exponentially in finite-time to the set $S=\left\{\sigma \in \mathbb{R}^{6}:\|\sigma\| \leq\|\mu\|(\right.$ or $\left.\|\delta\|)\right\}$ close to zero, from any initial condition $\|\sigma(0)\|>\|\mu\|$. This also implies that the tracking error converges exponentially in a finite-time $t_{f}$ to $S$, very close to zero and remains in $S \forall t>t_{f}$. Since in real-time applications, it is difficult for $\|\sigma\|$ to converge exponentially (in finite-time) to zero precisely, due to the measurement noise from the sensors (especially the depth and IMU sensors), sampled computation from the processors as well as the inherent nonlinear behaviour from the actuators.

\section{REAL-TIME EXPERIMENTAL RESULTS}

In this section, the scenarios-based real-time experimental results are presented and discussed. During the real-time implementation, different scenarios have been considered to evaluate the effectiveness of the proposed RAC law for realtime marine applications, as well as its robustness toward external disturbances.

\section{A. Some Implementation Issues}

Even though the controller proposed in this work has been designed for all the 6 DOFs, during the real-time experiments, we proposed to focus on the depth and yaw dynamics (2 DOFs) of the vehicle. The main goal of these experiments is to track the desired depth and yaw trajectories, as accurate as possible, in spite of the presence of external disturbances and parameter uncertainties. The real-time experiments have been conducted in a testing pool at LIRMM laboratory with a depth of about $1.2 \mathrm{~m}$. Where the proposed control scheme, designed in section III, is implemented using Visual $\mathrm{C}++$ on a computer (laptop) with Intel Core i7-3520M 2.9 GHz CPU, 8 GB of RAM and Windows 7 as operating system. Based on the signals acquired from the vehicle's depth and inertial measurement unit (IMU) sensors, the control law is computed for each DOF, then sent to the corresponding thruster (or thrusters) by the computer. The tested scenarios are described in the subsequent subsection and their video demonstration is available at: https : //www.youtube.com/watch?v = siMFn6pX $\mathbf{X}_{\mathrm{m}} \mathbf{Q}$.

\section{B. Proposed Experimental Scenarios}

1) Scenario 1 (External Disturbance Rejection): The objective of this test is to show the robustness of the proposed RAC in real-life applications. For instance, the robustness of the proposed RAC towards strong water current, sudden collision with marine animals (or structures in motion), tasks of carrying samples (or tools) and massive marine animal trying to reside on the vehicle. When an underwater vehicle using classical control comes across one of these situations, it can fail to track the desired trajectory. We approximate these situations with a heavy push applied to the Leonard ROV along the depth and yaw using a stick as illustrated in Fig. 3 Then, we evaluate the proposed RAC effectiveness to stabilise the vehicle around the desired trajectory.

2) Scenario 2 (Vehicle Buoyancy and Damping Change): In this scenario, the buoyancy and damping parameters of the vehicle are changed, as illustrated in Fig. 4. Then the robustness of the proposed RAC towards the parameters uncertainties is investigated.

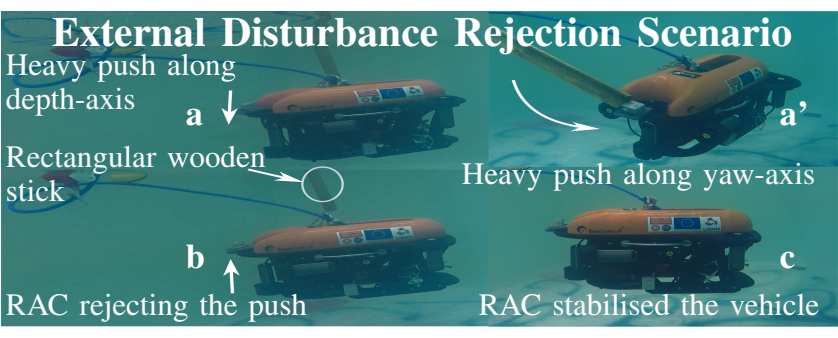

Fig. 3. Demonstration of external disturbance rejection scenario: (a-a') an external heavy push (20\% of the vehicle's weight) is applied to the depth and yaw axes. (b) external push removed. (c) the proposed RAC rejects the external push and stabilises the vehicle around the desired trajectory within 2 seconds when the heavy external push is removed.

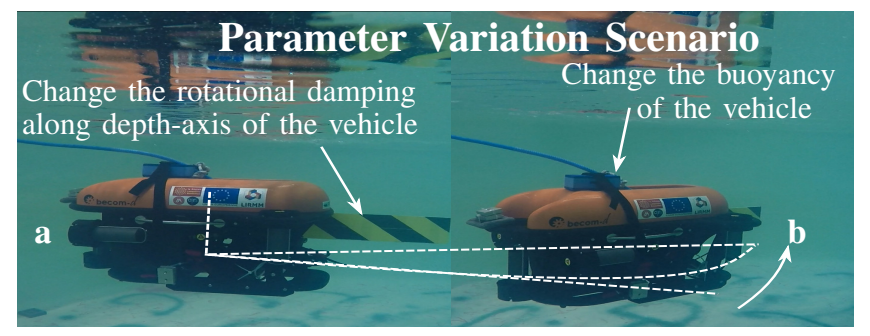

Fig. 4. Description of parameter variation scenario: (a) a rigid rectangular plastic sheet is attached to the vehicle. (b) floats are mounted on the surface of the vehicle. The rigid rectangular plastic sheet and the attached floats change the vehicle damping and buoyancy by approximately $90 \%$ and $>300 \%$, respectively.

\section{Control Parameters Tuning Method}

The proposed RAC parameters can be obtained as follows:

- assume a small $\left|\sigma_{i}\right|$, and design $\mu_{i}>\left|\sigma_{i}\right|$ for each DOF.

- if the bound of the uncertain parts of the system dynamics are known, choose $\Gamma_{0 i}>\left|\frac{\Phi_{b}}{\Omega_{b}}\right|$, otherwise assume $\Gamma_{0 i}$.

- increase $\Gamma_{i}$ slightly and observe the tracking error.

- decrease $\left|\sigma_{i}\right|$ to reduce the tracking error.

- for a system with a short sampling time $T_{s}$ and a known uncertainties bounds, $\mu_{i}=2 \Omega_{b} \cdot \Lambda_{i} \cdot T_{s}$, else $\mu_{i}=4 \cdot \Lambda_{i} \cdot T_{s}$

The proposed RAC parameters used during the proposed experiments are summarised in TABLE III.

\section{Scenario 1: Results}

This scenario is further subdivided into two cases as follows:

1) Case 1 ( $S 1-C 1)$ : In this test, the vehicle should follow a predefined reference trajectory, while a heavy push is applied along the depth axis of the vehicle using a rectangular wooden stick during the mission. The external push is applied when the vehicle is at $0.3 m$ depth for a period of 2 seconds, as shown in Fig. 5 (part-a of the top left plot). The magnitude of this push could be estimated at $25 \%$ of the weight of the

TABLE II

PROPOSED RAC CONTROLLER PARAMETERS

\begin{tabular}{|ll|l|l|l|}
\hline Depth & $\Lambda_{0 d}=1.000$ & $\Gamma_{d}=20.000$ & $\sigma_{0 d}=3.000$ & $\mu_{d}=0.010$ \\
& $\gamma_{d}=0.150$ & $\lambda_{0 d}=3.000$ & $\beta_{d}=0.300$ & $\delta_{d}=0.015$ \\
\hline Yaw & $\Lambda_{0 \psi}=1.000$ & $\Gamma_{\psi}=20.000$ & $\sigma_{0 \psi}=10.000$ & $\mu_{\psi}=0.010$ \\
& $\gamma_{\psi}=0.150$ & $\lambda_{0 \psi}=8.000$ & $\beta_{\psi}=0.100$ & $\delta_{\psi}=0.015$ \\
\hline
\end{tabular}


vehicle. The proposed RAC is able to reject the external push within about 2 seconds. We apply the same external push for the second time, while the vehicle is moving vertically from $0.3 m$ to $0.2 m$, as shown in Fig. 5 (part-b of the top left plot). Similarly, the proposed RAC is able to stabilise the vehicle for the second time, within about 2 seconds. To further evaluate the robustness of the proposed controller, we apply the same disturbance for the third time, when the vehicle is hovering at $0.2 \mathrm{~m}$. The robustness of the proposed controller is confirmed when the controller achieves to stabilise the vehicle for the third time within about 2 seconds again, as shown in Fig. 5 (part-c of the top left plot). The vehicle remains at $0.2 \mathrm{~m}$ until the end of this scenario, while the corresponding depth tracking error is shown in Fig. 5 (part-a', b' and c' of the top middle plot). Additionally, one can notice in Fig. 5 (part-a', b' and c' of the top middle plot) how our proposed controller is able to reject the effect of the external disturbance at every point of its application. Regarding the yaw tracking, the initial yaw is set to $-20^{\circ}$; thus allowing us to investigate the finitetime convergence of the yaw, as shown in Fig. 5 (bottom left plot). From Fig. 5 (bottom middle plot), we can notice a slight yaw tracking error even though the disturbance is applied to the depth. This tracking error is due to the vehicle's depth and yaw coupling dynamics. Besides, our proposed RAC is able to reject the external disturbance within a short period of time; there is a trade-off between the depth and yaw tracking errors with the energy consumption of the vehicle. Fig. 5 (top and bottom right plots) shows the evolution of both depth and yaw control inputs.

2) Case $2(S 1-C 2)$ : To further investigate the robustness of the proposed RAC towards external disturbance rejection with the external push applied along the yaw-axis, we propose to repeat the same process as in scenario $1(S 1-C 1)$. Our finding revealed more coupling effect when the external push is applied on the yaw axis, as shown in Fig. 6 (part-a, b, c and $\mathrm{d}$ of the top and bottom left plots). The tracking errors of both depth and yaw motions are shown in Fig. 6 (top and bottom middle plots). Additionally, we can observe a tracking error on the depth, even though the external push is applied along the yaw axis. This is because a slight linear displacement on the depth axis is caused by the rotational external push on the yaw axis. Therefore, a higher coupling effect is confirmed when the external disturbance is applied along the yaw axis. Finally, the evolution of the control efforts generated by the proposed controller for both depth and yaw trackings are depicted in Fig. 6 (top and bottom right plots).

\section{E. Scenario 2: Results}

The robustness of the proposed RAC approach towards uncertainties over the system parameters is investigated in this scenario. We increase the damping of the Leonard ROV along the yaw axis by $90 \%$ approximately from its nominal value. This modification is achieved by mounting a rigid rectangular $(45 \mathrm{~cm} \times 10 \mathrm{~cm})$ plastic sheet at the aft-most part of the vehicle (see Fig. 4). The plastic sheet creates an additional rotational drag when the vehicle turns along the yaw axis. Similarly, we modify the floatability of the vehicle by more than three times its default value using floats, as shown in Fig. 4 . Then, the vehicle should follow a similar trajectory as in previous scenarios. The obtained results are displayed in Fig. 7(top and bottom left plots). However, we doubled the initial yaw $\left(-40^{\circ}\right)$ of the vehicle, to reassess the finite-time convergence of the proposed control scheme from any initial state. The tracking errors evolution of both the depth and yaw is depicted in Fig. 7/(middle plots). Despite the modifications of the vehicle's parameters in this scenario, we can observe less yaw tracking error compared to the scenario $1(S 1-C 2)$ yaw tracking error, as summarised in TABLE III] However, TABLE III reveals less depth trackings errors of $S 1-C 1$ and $S 1-C 2$ in scenario 1 compared to the scenario $2(S 2)$ depth tracking error. Fig. 7 (top and bottom right plots) shows the control inputs needed to keep the vehicle very close to the desired trajectories.

\section{F. Comparison Study: Results}

The proposed RAC has been experimentally compared with the saturation based nonlinear PD control with gravity and buoyancy compensation $\left(N P D_{G B}\right)$, recently proposed in [10]; due to its robustness, its smaller computational cost, as well as its fast convergence rate. We repeated the scenario 2 $(S 2)$ described previously using the proposed RAC, and then $N P D_{G B}$, both implemented in real-time on Leonard ROV. From the obtained experimental results depicted in Fig. 8 (top left and top middle plots), our proposed RAC reduces the depth tracking offset with respect to $N P D_{G B}$ from $0.1120 m$ to $0.0390 m$ approximately (evaluated based on RMSE, criterion) resulting in $65 \%$ improvement. To be fair enough, we starts the experiment from the same initial condition for the depth and yaw as in [10]. However, we use a different initial condition for our proposed RAC in yaw tracking (i.e. $-40^{\circ}$, which is greater than in the $N P D_{G B}$ case) to rigorously rechallenge the controller proposed in this work. Our proposed RAC converges to the desired yaw within about 2 seconds, as shown in Fig. 8 (bottom left plot), which demonstrates finite-time convergence of the proposed RAC scheme. Even though both controllers are able to track the desired yaw with less than $0.1^{\circ}$ tracking error (i.e. $0.0799^{\circ}$ and $0.0117^{\circ}$ for $\mathrm{RAC}$ and $N P D_{G B}$ respectively, computed based on RMSE, criterion) tracking error, as shown in Fig. 8 (bottom middle plot). One can notice a slightly higher yaw tracking error of the proposed RAC. This error is due to the large initial condition, while converging faster to the desired yaw. The evolution of the control signals for both controllers is displayed in Fig. 8 (top and bottom right plots). It is worth to note from Fig. 8 (top right plot) that our proposed RAC demonstrates a better depth tracking performance while consuming $26 \%$ less energy than $N P D_{G B}$ on depth tracking (estimation based on integral of control inputs). However, one observes a higher energy consumption $40 \%$ compared to the $N P D_{G B}$ on the yaw tracking, due to the larger initial condition.

\section{CONCLusion ANd Future Work}

A Robust adaptive control (RAC) scheme has been proposed in this work. The proposed RAC approach has been implemented and validated in real-time on Leonard underwater 

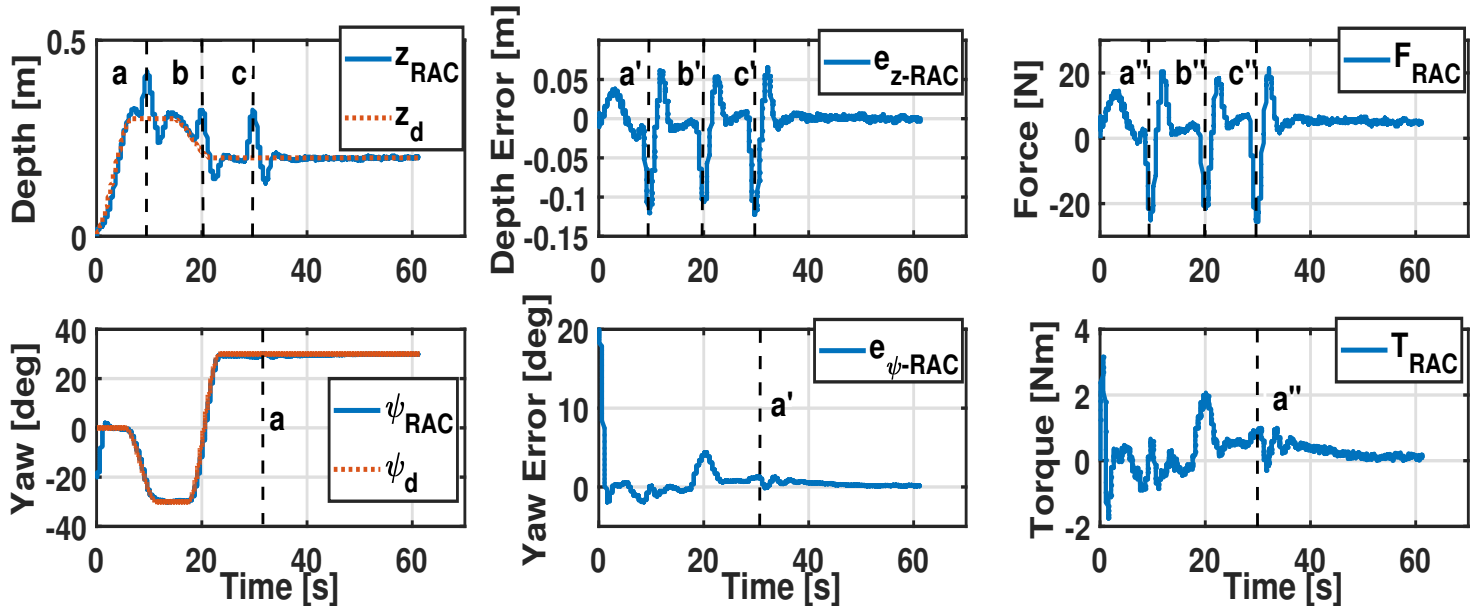

Fig. 5. External disturbance rejection case 1: (Left top and bottom plots) Robustness of the proposed RAC in rejecting an external disturbance applied along the depth-axis during tracking of the desired trajectory. (Middle top and bottom plots) Corresponding tracking errors in both depth and yaw. (Right top and bottom plots) Control inputs evolution.
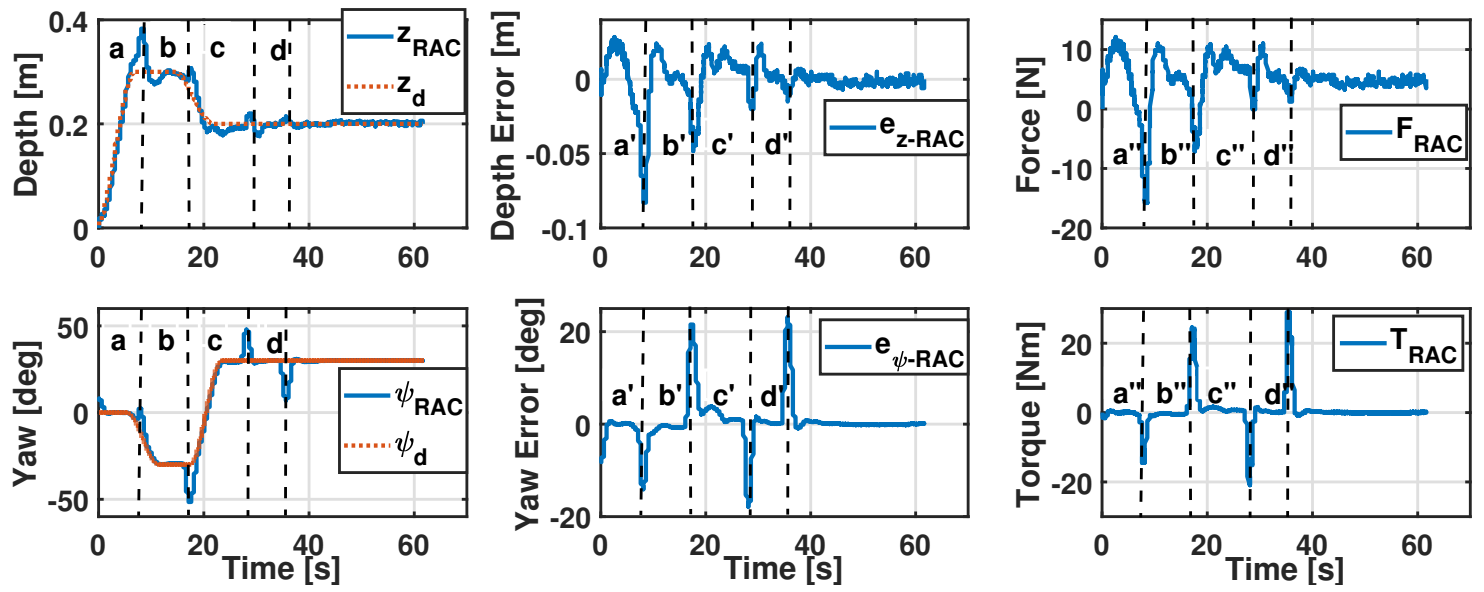

Fig. 6. External disturbance rejection case 2: (Left top and bottom plots) Robustness of the proposed RAC in rejecting an external disturbance applied along the yaw-axis during tracking of the desired trajectory. (Middle top and bottom plots) Trajectory tracking errors of the depth and yaw. (Right top and bottom plots) Control inputs evolution.
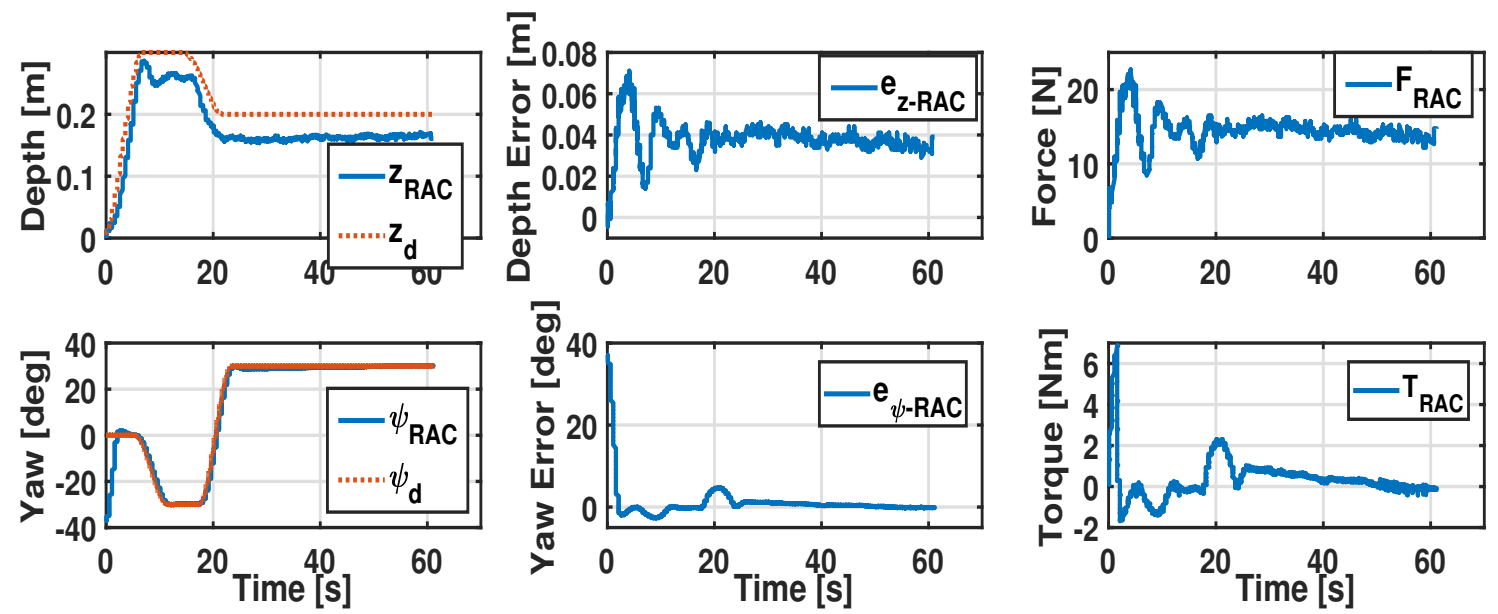

Fig. 7. Robustness of the proposed RAC towards parameter variations: A rigid rectangular plastic sheet and floats are attached to the robot. The rigid rectangular plastic sheet and the floats attached change the vehicle damping and buoyancy by approximately $90 \%$ and $>300 \%$, respectively. (Left plots) Depth and yaw trajectory tracking. (Middle plots) Tracking errors. (Right plots) Control inputs. 

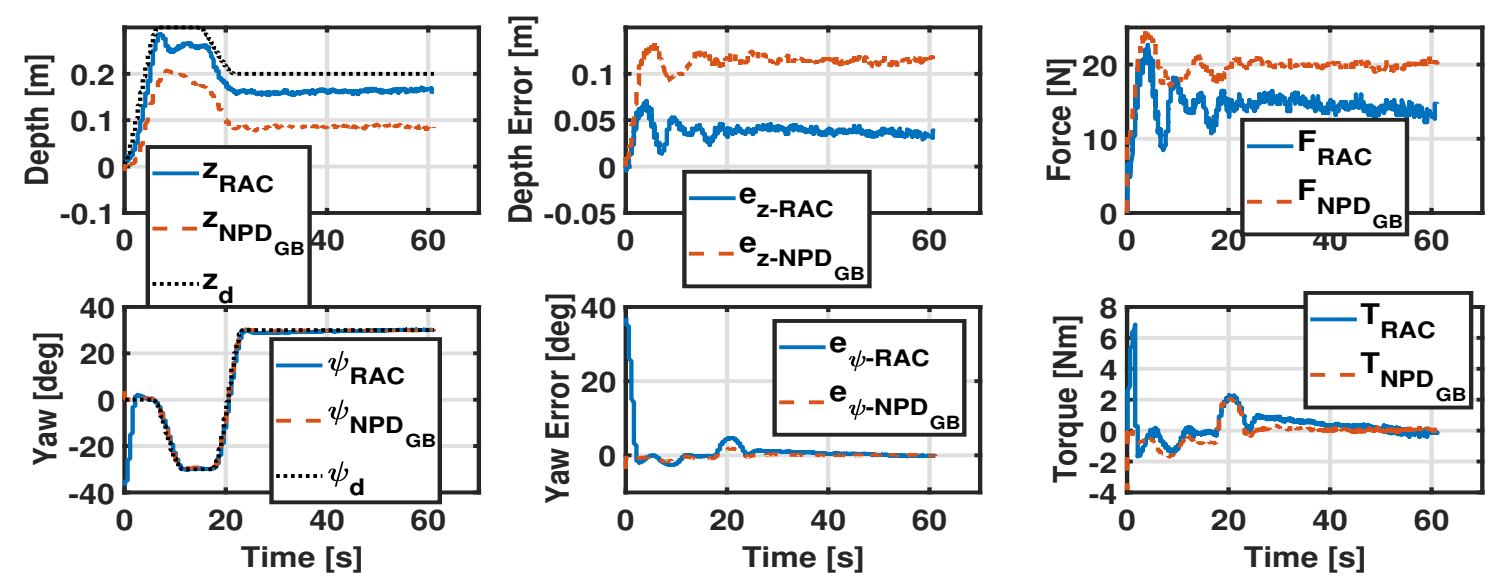

Fig. 8. Robustness comparison of the proposed RAC and $N P D_{G B}$ toward parameter variations: similar to scenario $S 2$, the vehicle damping and buoyancy were modified by $90 \%$ and $>300 \%$, respectively. (Left plots) Depth and yaw trajectory tracking. (Middle plots) Tracking errors. (Right plots) Control inputs.

TABLE III

RAC PERFORMANCE INDICES

\begin{tabular}{|ll|l|l|l|}
\hline & & RMSE & IAE & ISE \\
\hline Depth & $S 1-C 1$ & $3.19 \times 10^{-2}$ & $1.05 \times 10^{-0}$ & $6.14 \times 10^{-2}$ \\
& $S 1-C 2$ & $1.58 \times 10^{-2}$ & $5.71 \times 10^{-1}$ & $1.50 \times 10^{-2}$ \\
& $S 2$ & $3.90 \times 10^{-2}$ & $2.31 \times 10^{0}$ & $9.16 \times 10^{-2}$ \\
\hline Yaw & $S 1-C 1$ & $3.84 \times 10^{-2}$ & $9.56 \times 10^{-1}$ & $7.74 \times 10^{-1}$ \\
& $S 1-C 2$ & $9.08 \times 10^{-2}$ & $2.46 \times 10^{0}$ & $5.07 \times 10^{-1}$ \\
& $S 2$ & $7.99 \times 10^{-2}$ & $1.58 \times 10^{0}$ & $3.48 \times 10^{-1}$ \\
\hline
\end{tabular}

vehicle for autonomous trajectory tracking. Exponential stability and finite-time convergence of the resulting closed-loop tracking error to an invariant set, S (very close to zero) have been proven, based on Lyapunov arguments. Additionally, the proposed control scheme does not require prior any knowledge on the uncertainties bounds. This contrast with many of the proposed control schemes in the literature, where knowledge of these bounds is needed, and only asymptotic convergence is guaranteed. Scenarios-based real-time experimental results demonstrates the effectiveness and robustness of the proposed RAC scheme. The future work may focus on improving the vehicle tracking performance by integrating a continuousdiscrete time observer to estimate the uncertain parameters, as well as the external disturbances. Furthermore, we may also consider embedding an underwater robotic arm on the vehicle to enable more practical marine tasks (e.g. manipulation of underwater valves, collection of marine samples, etc.).

\section{APPENDIX A}

A. Based on the symmetric nature of the vehicle, $Z_{w} \approx Y_{v}$.

The following steps are used to obtain $Z_{w}$ : (i) Leonard is calibrated, and then the vehicle is actuated by a known thrusters forces in the z-direction. (ii) As the vehicle attends a steady speed in the $\mathrm{Z}$-direction, this speed is recorded. (iii) Then, $Z_{w}$ is estimated using the relationship: $Z_{w} \approx$ known force on $z$-direction calculated linear speed on $z$-direction.

Similarly, $X_{u}$ is estimated using distance covered by the vehicle along X-direction in the testing pool. $N_{r}$ is obtained using the same steps mentioned for estimating $Z_{w}$. However, during the process, linear velocity and known thrusters forces are replaced with angular rate from IMU reading and known thrusters torques respectively. Also $M_{q} \approx N_{r}$, while experimental estimation of $K_{p}$ required a vehicle design modification. As a result, $K_{p}$ has been estimated geometrically.

\section{B. For the restoring forces and moments (6):}

$$
F_{g(\eta)}=\left[\begin{array}{c}
(B-W) s \theta \\
(B-W) c \theta s \psi \\
(B-W) c \theta c \psi
\end{array}\right] \text { where B = buoyancy, } \mathrm{W}=m g \text { and }
$$

$M_{g(\eta)}=r_{c g} \times f_{W}^{v r f}+r_{c b} \times f_{B}^{v r f}$, with $r_{c g}$ and $r_{c b}$ are the center of gravity and buoyancy respectively.

\section{Equation (2) is transformed to (15) using:}

$$
\dot{\eta}=J(\eta) v, \ddot{\eta}=J(\eta) \dot{v}+\dot{J}(\eta) v, \bar{M}(\eta)=J^{-T}(\eta) M J^{-1}(\eta),
$$

$\bar{C}(v, \eta)=J^{-T}(\eta)\left[C(v)-M J^{-1}(\eta) \dot{J}(\eta)\right] J^{-1}(\eta)$,

$\bar{D}(v, \eta)=J^{-T}(\eta) D(v) J^{-1}(\eta), \bar{g}(\eta)=J^{-T}(\eta) g(\eta)$,

$\bar{\tau}(\eta)=J^{-T}(\eta) \tau$ and $\bar{w}^{\star}(t)=J^{-T}(\eta) w^{\star}(t)$.

\section{REFERENCES}

[1] N. Vedachalam, R. Ramesh, V. B. N. Jyothi, V. Doss Prakash, and G. Ramadass, "Autonomous underwater vehicles-challenging developments and technological maturity towards strategic swarm robotics systems," Marine Georesources \& Geotechnology, vol. 37, no. 5, pp. 525-538, 2019.

[2] E. Rusu, "A 30-year projection of the future wind energy resources in the coastal environment of the black sea," Renewable energy, vol. 139, pp. 228-234, 2019.

[3] A. Watt, M. R. Phillips, C.-A. Campbell, I. Wells, and S. Hole, "Wireless sensor networks for monitoring underwater sediment transport," Science of The Total Environment, 2019.

[4] D. Maalouf, A. Chemori, and V. Creuze, "L1 adaptive depth and pitch control of an underwater vehicle with real-time experiments," Ocean Engineering, vol. 98, pp. 66-77, 2015.

[5] R. A. S. Fernandez, Z. Milosevic, S. Dominguez, C. Rossi, et al., "Nonlinear attitude control of a spherical underwater vehicle," Sensors, vol. 19, no. 6, p. 1445, 2019.

[6] D. Ji, S. Zhou, J. Ren, and M. Sun, "A prototype of newly dynamic underwater vehicle using fuzzy pid control," in 2019 IEEE 28th International Symposium on Industrial Electronics (ISIE), pp. 1121-1126, IEEE, 2019.

[7] D. Lu, C. Xiong, Z. Zeng, and L. Lian, "Adaptive dynamic surface control for a hybrid aerial underwater vehicle with parametric dynamics and uncertainties," IEEE Journal of Oceanic Engineering, 2019. 
[8] R. M. Saback, A. G. S. Conceicao, T. L. M. Santos, J. Albiez, and M. Reis, "Nonlinear model predictive control applied to an autonomous underwater vehicle," IEEE Journal of Oceanic Engineering, 2019.

[9] X. Yang, J. Yan, C. Hua, and X. Guan, "Trajectory tracking control of autonomous underwater vehicle with unknown parameters and external disturbances," IEEE Transactions on Systems, Man, and Cybernetics: Systems, 2019.

[10] E. Campos, A. Chemori, V. Creuze, J. Torres, and R. Lozano, "Saturation based nonlinear depth and yaw control of underwater vehicles with stability analysis and real-time experiments," Mechatronics, vol. 45, pp. 49-59, 2017.

[11] J. Guerrero, J. Torres, V. Creuze, and A. Chemori, "Observationbased nonlinear proportional-derivative control for robust trajectory tracking for autonomous underwater vehicles," IEEE Journal of Oceanic Engineering, 2019.

[12] J. Guerrero, J. Torres, V. Creuze, A. Chemori, and E. Campos, "Saturation based nonlinear pid control for underwater vehicles: Design, stability analysis and experiments," Mechatronics, vol. 61, pp. 96-105, 2019.

[13] Y. Batmani and S. Najafi, "Event-triggered $\mathrm{h}^{\infty}$ depth control of remotely operated underwater vehicles," IEEE Transactions on Systems, Man, and Cybernetics: Systems, 2019.

[14] J. Guerrero, J. Torres, V. Creuze, and A. Chemori, "Trajectory tracking for autonomous underwater vehicle: An adaptive approach," Ocean Engineering, vol. 172, pp. 511-522, 2019.

[15] J. Cui, L. Zhao, J. Yu, C. Lin, and Y. Ma, "Neural network-based adaptive finite-time consensus tracking control for multiple autonomous underwater vehicles," IEEE Access, vol. 7, pp. 33064-33074, 2019.

[16] Z. Chen, C. Li, B. Yao, M. Yuan, and C. Yang, "Integrated coordinated/synchronized contouring control of a dual-linear-motor-driven gantry," IEEE Transactions on Industrial Electronics, 2019.

[17] M. U. Khalid, M. Ahsan, O. Kamal, and U. Najeeb, "Modeling and trajectory tracking of remotely operated underwater vehicle using higher order sliding mode control," in 2019 16th International Bhurban Conference on Applied Sciences and Technology (IBCAST), pp. 855-860, IEEE, 2019.

[18] B. Huang and Q. Yang, "Double-loop sliding mode controller with a novel switching term for the trajectory tracking of work-class rovs," Ocean Engineering, vol. 178, pp. 80-94, 2019.

[19] J. Kumar, V. Kumar, and K. Rana, "Design of robust fractional order fuzzy sliding mode pid controller for two link robotic manipulator system," Journal of Intelligent \& Fuzzy Systems, vol. 35, no. 5, pp. 5301$5315,2018$.

[20] J. de Jesús Rubio, A. Aguilar, J. A. Meda-Campaña, G. Ochoa, R. Balcazar, and J. Lopez, "An electricity generator based on the interaction of static and dynamic magnets," IEEE Transactions on Magnetics, vol. 55 , no. 8, pp. 1-11, 2019.

[21] L.-Y. Hao, H. Zhang, G. Guo, and H. Li, "Quantized sliding mode control of unmanned marine vehicles: Various thruster faults tolerated with a unified model," IEEE Transactions on Systems, Man, and Cybernetics. Systems, 2019.

[22] P. Herman, "Application of nonlinear controller for dynamics evaluation of underwater vehicles," Ocean Engineering, vol. 179, pp. 59-66, 2019.

[23] D. Wang, Y. Shen, Q. Sha, G. Li, X. Kong, G. Chen, and B. He, "Adaptive ddpg design-based sliding-mode control for autonomous underwater vehicles at different speeds," in 2019 IEEE Underwater Technology (UT), pp. 1-5, IEEE, 2019.

[24] A. Al-Mahturi, F. Santoso, M. A. Garratt, and S. G. Anavatti, "A robust adaptive interval type-2 fuzzy control for autonomous underwater vehicles," in 2019 IEEE International Conference on Industry 4.0, Artificial Intelligence, and Communications Technology (IAICT), pp. 1924, IEEE, 2019.

[25] E. Taheri, M. H. Ferdowsi, and M. Danesh, "Design boundary layer thickness and switching gain in smc algorithm for auv motion control," Robotica, vol. 37, no. 10, pp. 1785-1803, 2019.

[26] M. R. Ramezani-al and Z. Tavanaei-Sereshki, "An adaptive sliding mode controller with a new reaching law for tracking problem of an autonomous underwater vehicles," Transactions of the Institute of Measurement and Control, vol. 41, no. 6, pp. 1772-1787, 2019.

[27] A. Wadi, S. Mukhopadhyay, and J.-H. Lee, "A novel disturbancerobust adaptive trajectory tracking controller for a class of underactuated autonomous underwater vehicles," Ocean Engineering, vol. 189, p. 106377, 2019.

[28] X. Guo, W. Yan, and R. Cui, "Neural network-based nonlinear slidingmode control for an auv without velocity measurements," International Journal of Control, vol. 92, no. 3, pp. 677-692, 2019.
[29] Z. Dong, T. Bao, M. Zheng, X. Yang, L. Song, and Y. Mao, "Heading control of unmanned marine vehicles based on an improved robust adaptive fuzzy neural network control algorithm," IEEE Access, vol. 7, pp. 9704-9713, 2019

[30] Z. Chen, F. Huang, C. Yang, and B. Yao, "Adaptive fuzzy backstepping control for stable nonlinear bilateral teleoperation manipulators with enhanced transparency performance," IEEE transactions on industrial electronics, vol. 67, no. 1, pp. 746-756, 2019.

[31] X. Wang, G. Zhang, Y. Sun, J. Cao, L. Wan, M. Sheng, and Y. Liu, "Auv near-wall-following control based on adaptive disturbance observer," Ocean Engineering, vol. 190, p. 106429, 2019.

[32] J. de Jesús Rubio, "Robust feedback linearization for nonlinear processes control," ISA transactions, vol. 74, pp. 155-164, 2018.

[33] J. D. J. Rubio, G. Ochoa, D. Mujica-Vargas, E. Garcia, R. Balcazar, I. Elias, D. R. Cruz, C. F. Juarez, A. Aguilar, and J. F. Novoa, "Structure regulator for the perturbations attenuation in a quadrotor," IEEE Access, vol. 7, pp. 138244-138252, 2019.

[34] A. Marvian Mashhad and S. K.-e.-d. Mousavi Mashhadi, "Pd like fuzzy logic control of an autonomous underwater vehicle with the purpose of energy saving using $\mathrm{h}^{\infty}$ robust filter and its optimized covariance matrices," Journal of Marine Science and Technology, vol. 23, pp. 937949, 2018

[35] F. Plestan, Y. Shtessel, V. Bregeault, and A. Poznyak, "New methodologies for adaptive sliding mode control," International journal of control, vol. 83, no. 9, pp. 1907-1919, 2010.

[36] T. I. Fossen, "Guidance, navigation, and control of ships, rigs and underwater vehicles," Trondheim, Norway: Marine Cybernetics, 2002.

[37] Z. Guo, X. Yang, J. Gao, J. Yan, and X. Luo, "Velocity observer-based tracking control of autonomous underwater vehicle with communication delay," in 2019 3rd International Symposium on Autonomous Systems (ISAS), pp. 85-90, IEEE, 2019.

[38] R. Kelly, V. S. Davila, and J. A. L. Perez, Control of robot manipulators in joint space. Springer Science \& Business Media, 2006.

[39] J. Yan, J. Gao, X. Yang, X. Luo, and X. Guan, "Tracking control of a remotely operated underwater vehicle with time delay and actuator saturation," Ocean Engineering, vol. 184, pp. 299-310, 2019.

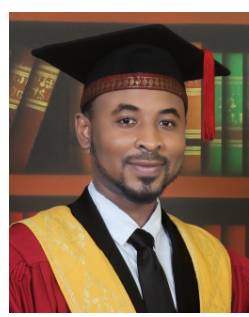

Auwal Shehu Tijjani received the B.ENG. and M.ENG. degrees from the Bayero University, Kano, Nigeria and Univesiti Teknologi Malaysia, Johor Bahru, Malaysia, in 2012 and 2016, respectively. $\mathrm{He}$ is currently a PhD Student at the University of Montpellier Laboratory of Informatics, Robotics, and Microelectronics. His research interests include robust and adaptive control of autonomous underwater vehicles, AUVs.

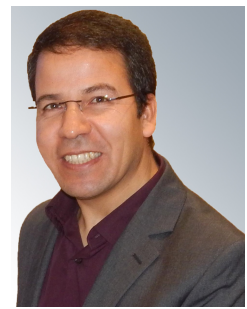

Ahmed CHEMORI received the M.Sc. and Ph.D degrees both in automatic control from the Grenoble Institute of Technology, Grenoble, France, in 2001 and 2005, respectively. He has been a Postdoctoral Fellow with the Automatic Control Laboratory, Grenoble, France, in 2006. He is currently a tenured Research Scientist in automatic control and robotics with the Montpellier Laboratory of Informatics, Robotics, and Microelectronics. His research interests include nonlinear, adaptive, and predictive control and their applications in robotics.

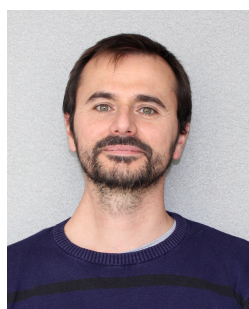

Vincent CREUZE received his $\mathrm{Ph} . \mathrm{D}$. degree in 2002 from the University Montpellier 2 (France). $\mathrm{He}$ is currently Associate Professor with Accreditation to Supervise Research, at the University of Montpellier, attached to the Robotics Department of the LIRMM. His research interests include design, modelling, sensing and control, applied to underwater robots for deep archaeological applications or marine biology. 